\title{
$L$-shell $\mathrm{x}$-ray production cross sections for light ions on $\mathrm{Sm}, \mathrm{Yb}$, and $\mathrm{Pb}^{\dagger} *$
}

\author{
Tom J. Gray, ${ }^{\ddagger}$ G. M. Light, R. K. Gardner, and F. D. McDaniel \\ Regional Nuclear Physics Laboratory, Department of Physics, North Texas State University, Denton, Texas 76203 \\ (Received 11 August 1975)

\begin{abstract}
$L$-shell x-ray production cross sections for the $L l, L \alpha_{1,2}, L \gamma_{1}$, and $L \gamma_{2,3,(6)}$ have been measured for ${ }^{1} \mathrm{H},{ }^{4} \mathrm{He}$, and ${ }^{7} \mathrm{Li}$ on $\mathrm{Yb}$ and $\mathrm{Pb}$ and for ${ }^{4} \mathrm{He}$ and ${ }^{7} \mathrm{Li}$ on $\mathrm{Sm}$. The energy ranges for the incident-ion species were 0.3 to $2.4 \mathrm{MeV} / \mathrm{amu}$ for ${ }^{1} \mathrm{H}, 0.15$ to $4.4 \mathrm{MeV} / \mathrm{amu}$ for ${ }^{4} \mathrm{He}$, and 0.9 to $3.0 \mathrm{MeV} / \mathrm{amu}$ for ${ }^{7} \mathrm{Li}$. Comparisons of the $L \alpha_{1,2}, L \gamma_{1}$, and $L \gamma_{2,3,(6)} \mathrm{x}$-ray production cross-section data have been made to the predictions of the plane-wave Born approximation (PWBA) and the PWBA with corrections for Coulomb deflection and/or binding-energy effects. The comparisons show that for the individual $x$-ray production cross sections, there are problems associated with the binding-energy correction calculations for the $L \gamma_{1}$ and $L \gamma_{2,3} \mathrm{x}$-ray data. Furthermore, measurements of the ratio of $L \alpha_{1,2}$ to $L \gamma_{2,3} \mathrm{x}$-ray intensities do not support the use of an increased effective binding energy in the PWBA formulation.
\end{abstract}

\section{INTRODUCTION}

Recent work on $L$-shell ionization by fast ions has been reported for thin-target geometries over energies ranging from $\sim 0.2$ to $\sim 4 \mathrm{MeV} / \mathrm{amu}$. Sev eral research groups ${ }^{1-7}$ have established the specific properties of the $\mathrm{x}$-ray production cross sections for protons and/or helium ions. Ratios of $\mathrm{x}$-ray cross sections have been analyzed to yield relative $\mathrm{x}$-ray cross-section data and the $L$-subshell ionization cross sections have been reported for protons on Au by Datz et al. ${ }^{1}$ The structure in the $L_{\mathrm{I}}$-subshell ionization cross section which arises from the $2 S$-character of the electrons in this subshell is well established. Comparisons of measured total $L$-shell $x$-ray cross sections to the plane-wave Born approximation $(\mathrm{PWBA})^{8}$ and the constrained-binary-encounter approximation (CBEA $)^{9}$ have shown that the se two theories gener ally agree with data for proton-induced $x$-ray cross sections.

In the measurements of $x$-ray cross-section ratios for protons on $\mathrm{Sm}^{2}$ and $\mathrm{Pr}, \mathrm{Eu}, \mathrm{Gd}$, and $\mathrm{Dy}^{2}$ the comparisons between the data and the PWBA and CBEA theories show that the PWBA is in much better agreement with the data for the ratio $\sigma_{L \alpha_{1,2}} / \sigma_{L \gamma_{2,3}}$ and $\sigma_{L \gamma_{1}} / \sigma_{L \gamma_{2,3}}$. The variations between the CBEA predictions and the data for these ratios can be related directly to a basic disagreement in the structure of the $L_{1}$-subshell ionization cross sections. It is suggested that the source of this disagreement is fundamental to the CBEA theory, possibly related to the velocity distribution of the $2 S$ electrons employed in this the ory. $^{2}$ The agreement between the CBEA and the $L_{\mathrm{II}}{ }^{-}$and $L_{\mathrm{III}}-$ related cross sections, which dominate the observed total $\mathrm{x}$-ray productions cross section, does not support arguments pertaining to multiple ionization as a causitive factor for the problems observed for the $2 S$ ionization.

When heavier ions such as ${ }^{4} \mathrm{He}$ ions are incident on a target, the PWBA calculations give $L$-shell $\mathrm{X}$-ray cross-section values which overestimate the observed magnitude of the total cross sections. Brandt and Lapicki ${ }^{10}$ have reported the results of including corrections to the PWBA. The corrections are suggested to account for the Coulomb deflection of the incident projectile through the ionnuclear interaction and to include perturbation ef fects on the electron binding energy due to the presence of the incident ion at the time of ionization. The net effect of these two corrections is to decrease the calculated cross section at low ion velocities. The corrections are dependent on the ion charge $Z_{1}$ as well as the ion velocity and target properties, the subshell binding energy $U_{\boldsymbol{L} \boldsymbol{i}}$, and the target atomic number $Z_{2}$.

The present paper presents studies of $L$-shell ionization by ${ }^{1} \mathrm{H},{ }^{4} \mathrm{He}$, and ${ }^{7} \mathrm{Li}$ on $\mathrm{Yb}$ and $\mathrm{Pb}$ and ${ }^{4} \mathrm{He}$ and ${ }^{7} \mathrm{Li}$ on $\mathrm{Sm}$ in the ion energy ranges of $\sim 0.3-$ $\sim 2.2 \mathrm{MeV} / \mathrm{amu}$ for ${ }^{1} \mathrm{H}, \sim 0.15 \sim \sim 4.4 \mathrm{MeV} / \mathrm{amu}$ for ${ }^{4} \mathrm{He}$, and $\sim 0.8 \sim 3.0 \mathrm{MeV} / \mathrm{amu}$ for ${ }^{7} \mathrm{Li}$. Comparisons to the PWBA and corrected PWBA theories were made to assess the effects of the Coulomb deflection and binding-energy corrections on the subshell ionization and $\mathrm{x}$-ray production cross sections.

\section{EXPERIMENTAL PROCEDURE AND DATA ANALYSIS}

For the low-energy work $(E<3.0 \mathrm{MeV})$ involving protons and helium ions, the 2.5-MV Van de Graaff accelerator of the Regional Nuclear Physics Laboratory at North Texas State University was employed. The experimental arrangement and procedure have been discussed in Ref. 2 for this facil ity. One modification to the arrangement has been effected in that the scattered ions for the nuclear 
elastic scattering are presently detected at a laboratory angle of $150^{\circ}$ in lieu of the $168^{\circ}$ angle used previously. The $\mathrm{x}$-ray detector efficiency is determined using calibrated reference sources as discussed previously. ${ }^{11}$ Thin targets of $\mathrm{SmF}_{3}$, $\mathrm{YbF}_{3}$, and $\mathrm{Pb}$ were prepared by vacuum evaporation of these materials on to thin carbon backings $\left(\rho x=20-50 \mu \mathrm{g} / \mathrm{cm}^{2}\right)$. Typical target thicknesses are less than $20 \mu \mathrm{g} / \mathrm{cm}^{2}$ for the materials studied.

The data for ion energies above $3 \mathrm{MeV}$ were taken using the Model EN tandem accelerator of the High Voltage Laboratory, Holifield National Laboratory. Ion beams of ${ }^{4} \mathrm{He}$ and ${ }^{7} \mathrm{Li}$ were obtained in the $2+$ and $3+$ charge states respectively. The beams were incident upon the targets positioned at an angle of $45^{\circ}$ with respect to the incident beam direction. X-ray and particle spectra were obtained with an ORTEC Si ( $\mathrm{Li}) \mathrm{x}$-ray detector having a resolution of $170 \mathrm{eV}$ at $5.89 \mathrm{keV}$ at a laboratory angle of $90^{\circ}$ and a silicon surface-barrier detector at a laboratory angle of $45^{\circ}$. The beam passed through a carbon collimator prior to strik ing the target to eliminate slit scattering from the main beam collimation system. Beam currents employed in the measurements were typically less than $10 \mathrm{nA}$. Count rates were limited to less than $1000 \mathrm{~Hz}$ in either counting channel (i.e., the $\mathrm{x}$-ray or nuclear elastic scattering channel). The data were accumulated in a Tennecomp data analysis system and transferred to magnetic tape for spectrum analysis using SAMPO. ${ }^{12}$

The $\mathrm{x}$-ray energy spectra were analyzed to give the yields for the $L \alpha_{1,2}, L \gamma_{1}$, and $L \gamma_{2,3,(6)} \mathrm{x}$-ray transitions. In the cases of Sm and $\mathrm{Yb}$ the $L \gamma_{2,3}$ transitions were analyzed. For $\mathrm{Pb}$ the $L \gamma_{6}$ transition is included as an unresolved component of the $L \gamma_{2,3,6} \mathrm{x}-$ ray line in the observed spectra. The $L \beta$ transitions were not analyzed as these observed $\mathrm{x}$-ray lines contain unresolved components which populate all three subshells. Consideration of the direct relationships between the $L \alpha_{1,2}, L \gamma_{1}$, and $L \gamma_{2,3,(6)} \times$-ray transitions and the $L_{\mathrm{II}^{-}}, L_{\mathrm{II}^{-}}$, and $L_{\mathrm{I}}$-subshell ionization processes, respectively, for incident ions shows that definitive measurements for the fundamentals of $L$-shell ionization by fast ions are obtained by experimental data for these three $\mathrm{x}$-ray transitions.

The measured $x$-ray intensities were converted to $\mathrm{x}$-ray production cross sections (XCS) for the $L \alpha_{1,2}, L \gamma_{1}$, and $L \gamma_{2,3}$ groups by normalization to the nuclear elastic scattering yields which were determined simultaneously at each incident ion energy. This procedure has been discussed previously. $^{2}$ The method of analysis utilized depends upon the nuclear elastic scattering cross section being described by Rutherford scattering. It has been shown that for ${ }^{7} \mathrm{Li}$ ions on $\mathrm{Rb}(Z=37)$ the observed elastic scattering at a laboratory angle of $45^{\circ}$ is non-Rutherford for incident energies above $21 \mathrm{MeV} .^{13}$ Consider the quantity

$$
\Gamma(E)=Y E^{2} / T_{R},
$$

where $Y$ is the measured nuclear elastic scattering yield, $E$ is the incident ion energy, and $T_{R}$ is the measured lifetime in the elastic scattering channel. $\Gamma(E)$ is a constant for Rutherford scattering. For the ion species on $\mathrm{Sm}, \mathrm{Yb}$, and $\mathrm{Pb}$ over the energy range of this work analyses of $\Gamma(E)$ confirms the use of the Rutherford scattering cross sections to describe the nuclear scattering probabilities.

In all cases the experimental XCS are presented. The comparisons to the theoretical predictions for the XCS are made by the use of the relationships discussed in Ref. 2. Table I lists the radiative and non-radiative parameters used in the theoretical calculations of the XCS for the $L \alpha_{1,2}, L \gamma_{1}$, and $L \gamma_{2,3,(6)} \mathrm{x}$-ray transitions. In all cases singlehole values for the parameters were used. The maximum observed energy shifts in the measured $\mathrm{X}$-ray transitions for ${ }^{7} \mathrm{Li}$ ions on $\mathrm{Sm}, \mathrm{Yb}$, and $\mathrm{Pb}$ were observed to be less than $15 \mathrm{eV}$. This suggests that multiple ionization is not a major effect to be considered in this work. Previous work by Pepper et al. ${ }^{14}$ for ${ }^{16} \mathrm{O}$ bombardment of the rare earths has shown that, even in those cases where multiple $M$-shell ionization dominates the observed $L$-shell radiations, the use of single-hole radiative and nonradiative parameters gives consistent results when the theories are compared to the experimental XCS data for $L$-shell ionization. Given that the target elements studied in this work are

TABLE I. Radiative and nonradiative rates used in the theoretical calculations for this work.

\begin{tabular}{rrr}
\hline \multicolumn{1}{c}{ Sm } & \multicolumn{1}{c}{ Yb } & \multicolumn{1}{c}{$\mathrm{Pb}$} \\
\hline$\omega_{1}=0.085^{\mathrm{a}}$ & $\omega_{1}=0.113^{\mathrm{a}}$ & $\omega_{1}=0.116^{\mathrm{a}}$ \\
$\omega_{2}=0.153^{\mathrm{a}}$ & $\omega_{2}=0.239^{\mathrm{a}}$ & $\omega_{2}=0.402^{\mathrm{a}}$ \\
$\omega_{3}=0.154^{\mathrm{a}}$ & $\omega_{3}=0.230^{\mathrm{a}}$ & $\omega_{3}=0.374^{\mathrm{a}}$ \\
$f_{12}=0.206^{\mathrm{a}}$ & $f_{12}=0.199^{\mathrm{a}}$ & $f_{12}=0.072^{\mathrm{a}}$ \\
$f_{13}=0.305^{\mathrm{a}}$ & $f_{13}=0.319^{\mathrm{a}}$ & $f_{13}=0.653^{\mathrm{a}}$ \\
$f_{23}=0.140^{\mathrm{a}}$ & $f_{23}=0.132^{\mathrm{a}}$ & $f_{23}=0.109^{\mathrm{a}}$ \\
$\Gamma_{1}=0.333^{\mathrm{b}}$ & $\Gamma_{1}=0.608^{\mathrm{b}}$ & $\Gamma_{1}=1.349^{\mathrm{b}}$ \\
$\Gamma_{1 \gamma 2}=0.024^{\mathrm{b}}$ & $\Gamma_{1 \gamma 2}=0.047^{\mathrm{b}}$ & $\Gamma_{1 \gamma_{2}}=0.117^{\mathrm{b}}$ \\
$\Gamma_{1 \gamma 3}=0.037^{\mathrm{b}}$ & $\Gamma_{1 \gamma 3}=0.066^{\mathrm{b}}$ & $\Gamma_{1 \gamma_{3}}=0.139^{\mathrm{b}}$ \\
$\Gamma_{2}=0.568^{\mathrm{b}}$ & $\Gamma_{2}=1.051^{\mathrm{b}}$ & $\Gamma_{2}=2.378^{\mathrm{b}}$ \\
$\Gamma_{2 \gamma 1}=0.080^{\mathrm{b}}$ & $\Gamma_{2 \gamma 1}=0.154^{\mathrm{b}}$ & $\Gamma_{2 \gamma_{1}}=0.383^{\mathrm{b}}$ \\
$\Gamma_{3}=0.524^{\mathrm{b}}$ & $\Gamma_{3}=0.947^{\mathrm{b}}$ & $\Gamma_{2 \gamma}=0.047^{\mathrm{b}}$ \\
$\Gamma_{3 \alpha 1}=0.386^{\mathrm{b}}$ & $\Gamma_{3 \alpha_{1}}=0.693^{\mathrm{b}}$ & $\Gamma_{3}=2.060^{\mathrm{b}}$ \\
$\Gamma_{3 \alpha_{2}}=0.044^{\mathrm{b}}$ & $\Gamma_{3 \alpha_{2}}=0.078^{\mathrm{b}}$ & $\Gamma_{3 \alpha_{1}}=1.438^{\mathrm{b}}$ \\
& & $\Gamma_{3 \alpha_{2}}=0.163^{\mathrm{b}}$ \\
\hline \hline
\end{tabular}

\footnotetext{
${ }^{\mathrm{a}}$ Reference 16 .

${ }^{\mathrm{b}}$ Reference 17 .
} 
similar in their characteristics while the incident ion species are lighter than ${ }^{16} \mathrm{O}$, it is expected that a similar type of analysis will prevail. This assumption must be considered as a possible source of error until more detailed information may be available through either higher-resolution studies or further theoretical studies.

Error analysis for the experimental data includes two main categories of error: (i) the relative uncertainty which consists of statistical errors associated with the individual $x$-ray yields and charged particle elastic scattering $(2-10 \%$ and $1-3 \%$, respectively) and (ii) the overall normalization uncertainty which contains contributions from calibrated source strength error $(3 \%)$, x-ray source branching ratio uncertainties (1-2\%), chargedparticle-detector solid-angle error (3\%), and uncertainty in Rutherford scattering cross section through detector angle $(<5 \%)$. The overall normalization error is obtained by taking the root mean square of the individual contributions giving an error of $<8 \%$. In the relative uncertainties the $L \alpha_{1,2} \mathrm{x}$-ray transitions have errors of $<3 \%$ while the $L \gamma_{1}$ and $L \gamma_{2,3,(6)}$ transitions have errors of $<11 \%$. The total uncertainties are taken as $<9 \%$ for the $L \alpha_{1,2}$ lines and $<14 \%$ for the $L \gamma_{1}$ and $L \gamma_{2,3,(6)}$ lines.

\section{DISCUSSION}

Shown in Figs. 1, 2, and 3 are the measured $L \alpha_{1,2}$, $L \gamma_{1}$, and $L \gamma_{2,3,(6)} \mathrm{XCS}$, respectively, for ${ }^{1} \mathrm{H},{ }^{4} \mathrm{He}$, and ${ }^{7} \mathrm{Li}$ on $\mathrm{Yb}$ and $\mathrm{Pb}$ and for ${ }^{4} \mathrm{He}$ and ${ }^{7} \mathrm{Li}$ on $\mathrm{Sm}$. In each case the experimental data are compared to the predictions of the plane-wave Born approximation (PWBA) of Choi, Merzbacher, and Khandelwal ${ }^{8}$ and the PWBA with corrections for binding energy and Coulomb deflection effects (PWBABC) by Brandt and Lapicki. ${ }^{10}$ It is observed for the $L \alpha_{1,2} \mathrm{XCS}$, as shown in Fig. 1, that the PWBABC is in better agreement with the data for $\mathrm{Sm}$ and $\mathrm{Yb}$ for each incident ion species. In the case of $\mathrm{Pb}$ the data for $L \alpha_{1,2}$ begins to deviate from the PWBABC for ion energies $\leqslant 1.2 \mathrm{MeV} / \mathrm{amu}$, with the data being larger in magnitude than the PWBABC predictions. The PWBA overpredicts the observed $L \alpha_{1,2}$ XCS data in all cases. The deviations observed for the $\mathrm{Pb}$ case may be associated with the need for a relativistic description for the $L$-shell electrons in $\mathrm{Pb}$ as suggested by Choi. ${ }^{15}$ The present calculations do not include such considerations. The comparison of the PWBABC to the $L \alpha_{1,2}$ data of this work for Sm, $\mathrm{Yb}$, and $\mathrm{Pb}$ are in excellent agreement with similar comparisons given by Brandt and Lapicki for the $L$-shell ionization data for protons on $\mathrm{Au}$ of Datz et $a l .^{1}$
The experimental measurements for the $L \gamma_{1} \mathrm{XCS}$ from this work are given in Fig. 2. Comparisons to the PWBA and PWBABC predictions are given for these data. A study of the measurements shows that in contrast to the results for the $L \alpha_{1,2}$ data the PWBA calculations give a better representation of the $L \gamma_{1}$ cross-section magnitudes except for the lower ion energies. In the case of ${ }^{1} \mathrm{H}$ the data begin to deviate from the PWBA predictions for $E \leqslant 0.5$ $\mathrm{MeV} / \mathrm{amu}$ for $\mathrm{Yb}$ and $\mathrm{Pb}$. In the case of ${ }^{4} \mathrm{He}$ bombardment the PWBA predictions are in better agreement with the data over the energy range studied. Under ${ }^{7} \mathrm{Li}$ bombardment the agreement be tween the data and PWBA predictions for the $L \gamma_{1}$ $\mathrm{XCS}$ tends to improve with the increase in atomic number of the target which may again be due to relativistic effects. ${ }^{15}$

As observed in Fig. 3 the $L \gamma_{2,3,(6)}$ XCS data for ${ }^{1} \mathrm{H},{ }^{4} \mathrm{He}$, and ${ }^{7} \mathrm{Li}$ on $\mathrm{Yb}, \mathrm{Pb}$, and $\mathrm{Sm}\left({ }^{4} \mathrm{He}\right.$ and ${ }^{7} \mathrm{Li}$ only) shows the same general trends as the $L \gamma_{1}$ data. The PWBA predictions give a better overall representation of the measured $L \gamma_{2,3,(6)}$ XCS data except for the lower bombarding energies. Clearly, the PWBABC calculations are not adequate in their

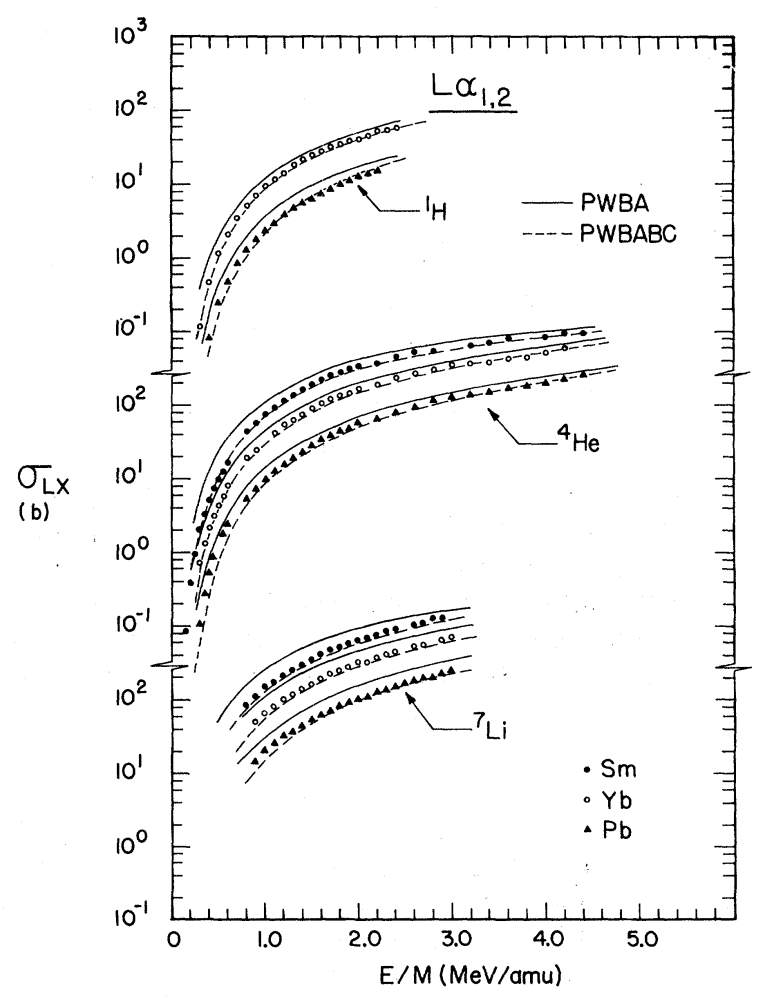

FIG. 1. Experimental $x$-ray production cross sections for the $L \alpha_{1,2}$ transition. The PWBA calculations are from Ref. 8 and the PWBABC predictions are from Ref. 10 . 
description of the $L \gamma_{1}$ and $L \gamma_{2,3,(6)}$ XCS data for $E / m \gtrsim 0.8 \mathrm{MeV} / \mathrm{amu}$. The results of this work for the transitions, which are related to the $L_{\mathrm{II}^{-}}$and $L_{\mathrm{I}}$-subshell ionization processes, are closely correlated to similar results obtained by Brandt and Lapicki ${ }^{10}$ for the Au $L$-subshell data of Datz et al. Brandt and Lapicki suggest that uncertainties in the radiative and nonradiative parameters may be responsible for the deviations between the PWBABC and the $L_{\mathrm{I}}$ - and $L_{\mathrm{II}}$-subshell ionization data for Au. While this suggestion may have some validity, there are problems, associated with the binding-energy correction, which are independent of the radiative and nonradiative parameters if it is assumed that these quantities do not change significantly as a function of ion energy for ${ }^{1} \mathrm{H}$, ${ }^{4} \mathrm{He}$, and ${ }^{7} \mathrm{Li}$ on elements having atomic numbers in the range of this work. High-resolution work for ${ }^{16} \mathrm{O}$ on La by Pepper et al. ${ }^{14}$ has shown significiant $M$-shell multiple ionization accompanying the $L$-shell ionization process. However, no double $L$-shell ionization was observed. In the absence of multiple $L$-shell vacancies the CosterKronig rates should be essentially constant. While multiple ionization is to be expected to some degree for ${ }^{7} \mathrm{Li}$ and in decreasing contributions for

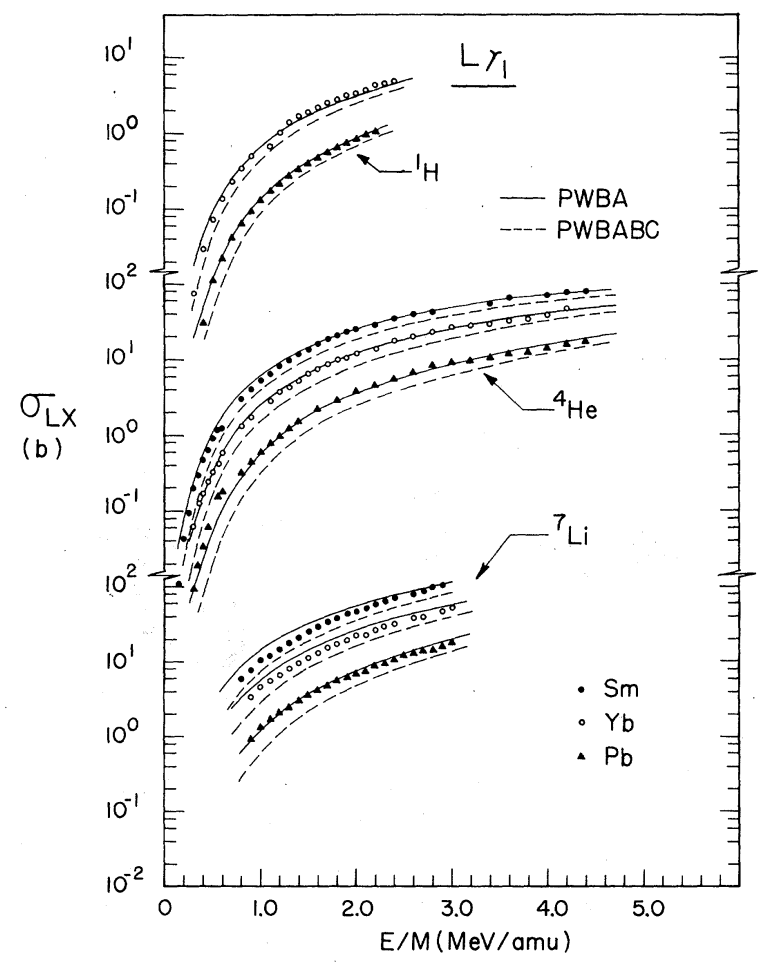

FIG. 2. Experimental $L \gamma_{1}$ x-ray production cross sections.
${ }^{4} \mathrm{He}$ and ${ }^{1} \mathrm{H}$, its effects on the observed XCS data should be less than observed for ${ }^{16} \mathrm{O}$ bombardment.

Calculations for the individual contributions for the binding-energy corrections (BE) and Coulomb deflection corrections (CD) to the PWBABC predictions are shown for ${ }^{1} \mathrm{H},{ }^{4} \mathrm{He}$, and ${ }^{7} \mathrm{Li}$ on $\mathrm{Yb}$ in Fig. 4. In the case of the $L \alpha_{1,2} \mathrm{XCS}$ data it is observed that the PWBA (CD) is not sufficient by it self to give the proper magnitude estimates in comparison to the data. For ${ }^{4} \mathrm{He}$ and ${ }^{7} \mathrm{Li}$ the PWBA (BE) represents the majority of the PWBABC predictions. However, in the comparisons to the $L \gamma_{1}$ and $L \gamma_{2,3},(6)$ data the PWBA (BE) calculations are seen to be the source of the disagreement between the PWBABC and the XCS data for the $L \gamma_{1}$ and $L \gamma_{2,3,(6)}$ transitions. Furthermore, in the case of the $L \gamma_{2,3,(6)}$ data it is observed that the PWBA $(\mathrm{BE})$ calculations cause a shift in the structure in the cross sections which is related to the $2 S$ character of the $L_{\mathrm{I}}$ subshell. This feature is particularly clear for the ${ }^{4} \mathrm{He}$ data as shown in Fig. 4 .

Analysis of the data for the ratio of the $L \alpha_{1,2}$ and $L \gamma_{2,3,(6)}$ cross sections is shown in Fig. 5 . For each target species, $\mathrm{Sm}, \mathrm{Yb}$, and $\mathrm{Pb}$, it is observed that for ${ }^{1} \mathrm{H},{ }^{4} \mathrm{He}$, and ${ }^{7} \mathrm{Li}$ the PWBA correctly predicts the shape of the peak in the ratio

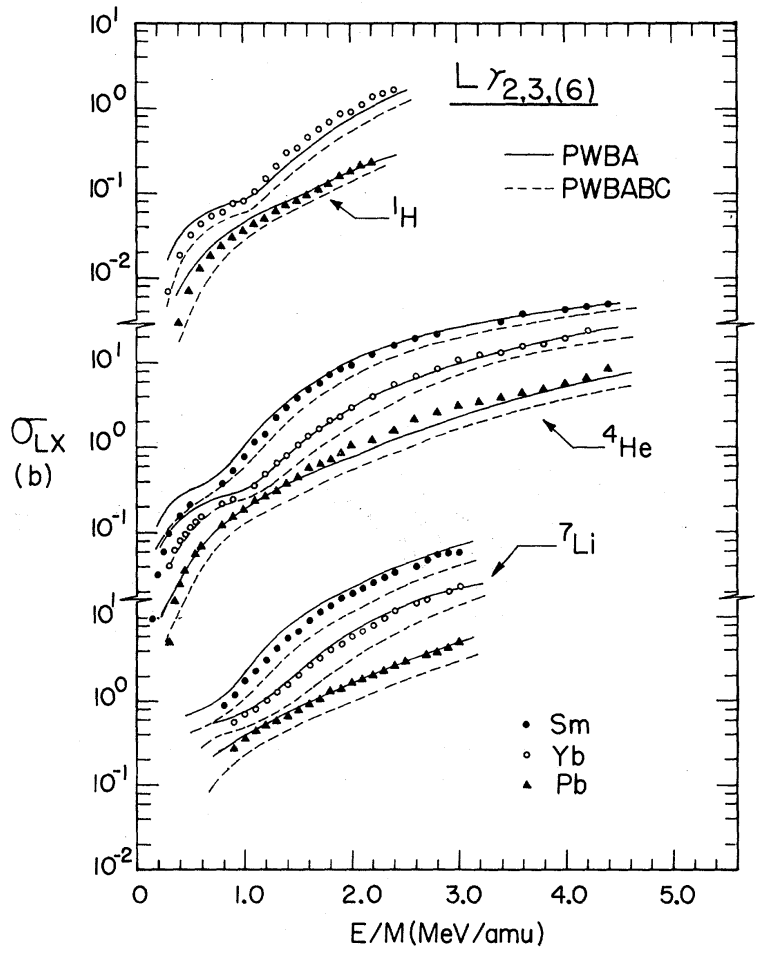

FIG. 3. Experimental $L \gamma_{2,3}$ x-ray production cross sections. 
$R$, where

$$
R=\sigma_{L \alpha_{1,2}} / \sigma_{L \gamma_{2,3,(6)}} \cdot
$$

The PWBABC calculations cause the peak in the predicted ratio to move to higher $E / M$ values as the incident ion $Z$ is increased from ${ }^{1} \mathrm{H}$ to ${ }^{4} \mathrm{He}$ to ${ }^{7} \mathrm{Li}$. The Coulomb deflection correction does not cause a shift in $R$ with increasing incident ion species. The binding-energy correction is obtained by increasing the individual subshell binding energies to account for the presence of the ion during the ionization process. The use of an increased effective binding energy naturally leads to the calculated shift in the $2 S$-related structure in the $L \gamma_{2,3,(6)}$ cross-section data.

\section{CONCLUSIONS}

It is concluded that the observed $L$-shell $\mathrm{x}$-ray production cross sections for ${ }^{1} \mathrm{H}$, ${ }^{4} \mathrm{He}$, and ${ }^{7} \mathrm{Li}$ on $\mathrm{Sm}, \mathrm{Yb}$, and $\mathrm{Pb}^{18}$ can be represented with varying degrees of agreement by the PWBABC or PWBA theories. One theory is not sufficient to give the

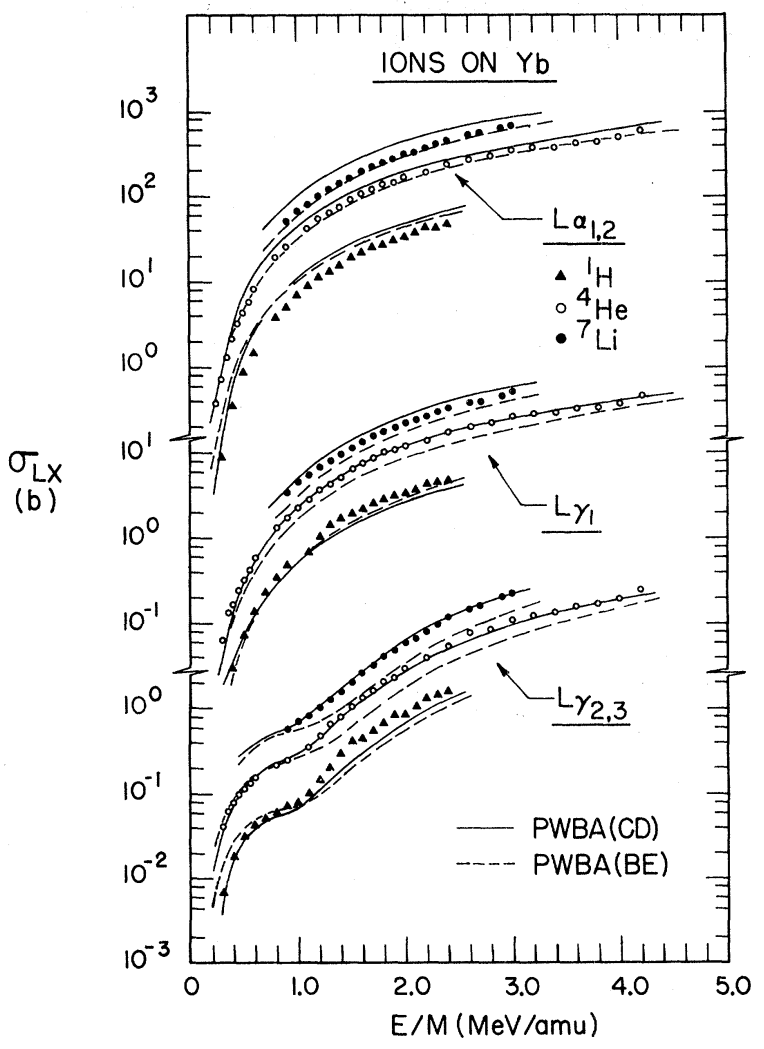

FIG. 4. Experimental $L \alpha_{1,2}, L \gamma_{1}$, and $L \gamma_{2,3} \mathrm{x}$-ray production cross sections for $\mathrm{Yb}$ compared to the PWBA predictions with Coulomb deflection (CD) and bindingenergy (BE) corrections applied separately. The CD and $\mathrm{BE}$ corrections are from Ref. 10. best overall agreement with the data for all three $L$ subshells as represented by the $L \alpha_{1,2}, L \gamma_{1}$, and $L \gamma_{2,3,(6)} \mathrm{X}$-ray transitions. While the PWBABC approach does give a much better representation of the $L \alpha_{1,2}$ data for the ion species of this work in comparison to the PWBA theory, there are problems with the static effective binding-energy increase as utilized in the PWBABC calculations. PWBABC predictions for the $L \gamma_{1}$ and $L \gamma_{2,3,(6)} \mathrm{X}-$ ray cross sections and the energy shift in the $2 S$ related structure in the $L \gamma_{2,3,(6)}$ cross section, as required by the PWBABC approach, are not supported by the data of this work. The main causative factor in such disagreements is found in the binding-energy correction of the PWBABC. The uncertainties in the radiative ${ }^{19}$ and nonradiative parameters which are required for comparisons between the data and the theory may be a source of error in the predicted magnitudes, but it is not plausible that such uncertainties should be related solely to the $L_{\mathrm{II}}$ and $L_{\mathrm{T}}$-subshell processes. It is suggested that the problems associated with the lack of an energy shift in the $L \gamma_{2,3,(6)}$ cross-section data raises a serious question about the validity of a fixed average binding -energy increase as a viable approximation to a perturbation effect

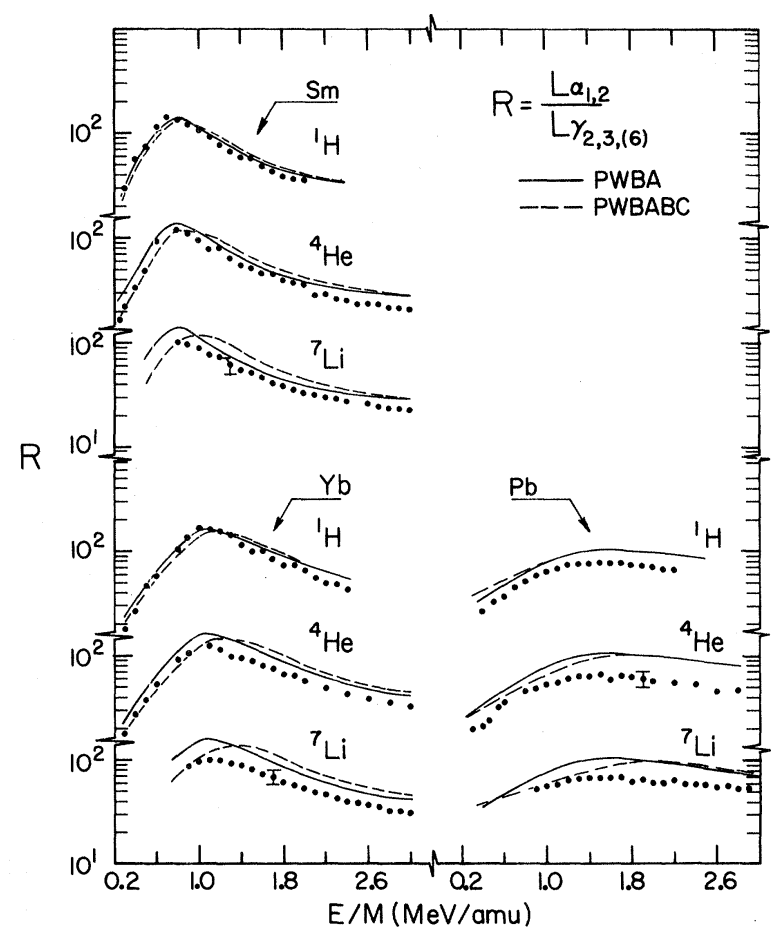

FIG. 5. Ratios of the $L \alpha_{1,2}$ and $L \gamma_{2,3,(6)} \mathrm{x}$-ray production cross sections compared to the PWBA and PWBABC predictions. The peak positions in the experimental ratios are constant to first order for each element as the incident ion species are varied from ${ }^{1} \mathrm{H}$ to ${ }^{7} \mathrm{Li}$. The data for ${ }^{1} \mathrm{H}$ on Sm are from Ref. 2. 
which one may expect to exist. It would seem appropriate to engage in further theoretical studies which may provide for the inclusion of such a perturbation correction as the binding-energy effect in a more dynamical way.

\section{ACKNOWLEDGMENTS}

The authors would like to thank J. L. Duggan, R. L. Wheeler, and A. R. Zander for their assis- tance with data acquisition during the high-energy portions of the present work. We graciously acknowledge the assistance of the entire staff of the Tandem Accelerator Laboratory at the Holifield National Laboratory and especially the assistance of J. A. Biggerstaff and C. D. Moak. We are especially grateful to G. H. Pepper for the use of the computer code XCODE which was employed for the theoretical calculations of this work. $\dagger$ Work supported in part by the Robert A. Welch Foundation, the Faculty Research Fund (NTSU), and Oak Ridge Associated Universities.

*Work performed in part on the HVEC tandem accelerator, Holifield National Laboratory, under support by the United States Energy Research and Development Administration.

\$Present address: Department of Physics, Kansas State University, Manhattan, Kansas 66506 , on leave from North Texas State University.

${ }^{1}$ S. Datz, J. L. Duggan, L. C. Feldman, E. Laegsgaard, and J. V. Andersen, Phys. Rev. A 9 , 192 (1974).

${ }^{2}$ F. Abrath and Tom J. Gray, Phys. Rev. A $\underline{9}, 682$ (1974); 10, 1157 (1974).

${ }^{3}$ D. H. Madison, A. B. Baskin, C. E. Busch, and S. M. Shafroth, Phys. Rev. A $\underline{9}, 675$ (1974).

${ }^{4}$ S. M. Shafroth, G. A. Bissinger, and A. W. Waltner, Phys. Rev. A $\underline{7}, 566$ (1973).

${ }^{5}$ H. Tawara, K. Ishii, S. Morita, H. Kaji, and T. Shiokawa, Phys. Rev. A 11, 1560 (1975).

${ }^{6}$ C. N. Chang, J. F. Morgan, and S. L. Blatt, Phys. Rev. A 11, 607 (1975).

${ }^{7}$ C. E. Busch, A. B. Baskin, P. H. Nettles, S. M. Shaf- roth, and A. W. Waltner, Phys. Rev. A 7, 1601 (1973). ${ }^{8}$ B.-H. Choi, E. Merzbacher, and G. S. Khandelwal, At. Data $\underline{5}, 291$ (1973).

${ }^{9}$ J. S. Hansen, Phys. Rev. A 8,822 (1973).

${ }^{10}$ W. Brandt and G. Lapicki, Phys. Rev. A 10, 474 (1974).

${ }^{11}$ R. Lear and Tom J. Gray, Phys. Rev. A $\underline{8}, 2469$ (1973).

${ }^{12} \mathrm{~J}$. T. Routti and S. G. Prussin, Nucl. Instrum. Methods 72,125 (1969).

${ }^{13}$ F. D. McDaniel, Tom J. Gray, R. K. Gardner, G. M. Light, J. L. Duggan, H. A. Van Rinsvelt, R. D. Lear, G. H. Pepper, J. William Nelson, and Arlen R. Zander, Phys. Rev. A 12, 1271 (1975).

${ }^{14}$ G. H. Pepper, R. D. Lear, Tom J. Gray, R. P. Chaturvedi, and C. Fred Moore, Phys. Rev. A 12, 1237 (1975). ${ }^{15}$ B.-H. Choi, Phys. Rev. A 4 , 1002 (1971).

${ }^{16}$ E. J. McGuire, Phys. Rev. A $\underline{3}, 587$ (1971).

${ }^{17}$ J. H. Scofield, Phys. Rev. 179,9 (1969).

${ }^{18}$ Tabulated values for the $\mathrm{x}$-ray production cross sections are available from Tom J. Gray.

${ }^{19} \mathrm{It}$ is noted that the $L$-shell radiative widths from J. $H$. Scofield [Phys. Rev. A 10, 1507 (1974)], differ from the values of Ref. 17 by $\sim 1-3 \%$, which is within the quoted experimental errors of the present work. 